\title{
Boldo-Induced Hepatotoxicity: A Case of Unexplained Jaundice
}

\author{
Ana Oliveira Sá, Teresa Pimentel, Narciso Oliveira \\ Hospital de Braga, Braga, Portugal
}

Doi: 10.12890/2020_002116 - European Journal of Case Reports in Internal Medicine - ๑ EFIM 2020

Received: 04/11/2020

Accepted: $10 / 11 / 2020$

Published: $27 / 11 / 2020$

How to cite this article: Sá A, Pimentel T, Oliveira N. Boldo-induced hepatotoxicity: a case of unexplained jaundice. EJCRIM 2020;7: doi:10.12890/2020_002116.

Conflicts of Interests: The Authors declare that there are no competing interests.

This article is licensed under a Commons Attribution Non-Commercial 4.0 License

\section{ABSTRACT}

An 87-year-old man was admitted with a 2-week history of asthenia, anorexia and jaundice. Blood tests showed increased serum liver enzymes. The aetiological study was inconclusive. The patient had a favourable clinical evolution during hospitalization with supportive care. His wife revealed that the patient had repeatedly consumed infusions of Peumus boldus (commonly known as boldo) leaves during the previous month. After common causes of hepatobiliary pathology were excluded, boldo-induced hepatotoxicity was considered probable. Cessation of its consumption led to complete clinical and laboratory recovery within a week.

\section{LEARNING POINTS}

- Herbal medicines are commonly used by the public.

- Peumus boldus leaves consumed as an infusion can be hepatotoxic and may cause otherwise unexplained jaundice or abnormal liver enzyme values, particularly in elderly patients.

- A detailed clinical history is crucial to establish the consumption of alternative medicines in order to identify possible adverse effects and toxicity.

\section{KEYWORDS}

Peumus boldus, herbal medicines, hepatotoxicity

\section{CASE DESCRIPTION}

We report the case of an 87-year-old Caucasian man with arterial hypertension and polymyalgia rheumatica treated with perindopril/ indapamide 4/1.25 mg, prednisolone $25 \mathrm{mg}$ and pantoprazole $20 \mathrm{mg}$. He was admitted to hospital with a 2-week history of asthenia, anorexia and jaundice. He denied having abdominal pain or fever. He also denied consumption of contaminated food, changes in diet or medication, recent travel, or contact with toxic products or with animals.

His physical examination was unremarkable except for severe dehydration and jaundice. Laboratory tests showed increased total bilirubin (3.42 mg/dl), conjugated bilirubin (1.06 mg/dl), aspartate transaminase (1455 U/l), alanine aminotransferase (1044 U/l), alkaline phosphatase (248 U/I), gamma-GT (683 U/I), amylase (204 U/I) and lipase (1706 U/I). Serum protein electrophoresis was normal. Renal function, an ionogram and the remaining biochemical parameters were also normal. The hepatitis A, B and C, cytomegalovirus and EpsteinBarr serologies and the VDRL were all negative. Immunological tests were negative. No drugs or alcohol were present. An abdominal CT scan and MRI showed only a known unspecific subcapsular cyst about $4 \mathrm{~cm}$ in size in the upper posterior margin of the right lobe. Upper GI endoscopy was normal. The patient was given supportive care.

A conversation with his wife revealed he had repeatedly consumed an infusion of Peumus boldus (commonly known as boldo) leaves for the past month due to dyspepsia (Fig. 1). After exclusion of common causes of hepatobiliary pathology, boldo-induced hepatotoxicity was 
considered probable. Cessation of its ingestion led to complete clinical and laboratory recovery within a week. After a year of follow-up without consumption of Peumus boldus, the patient is asymptomatic and serum liver enzymes remain within normal values.

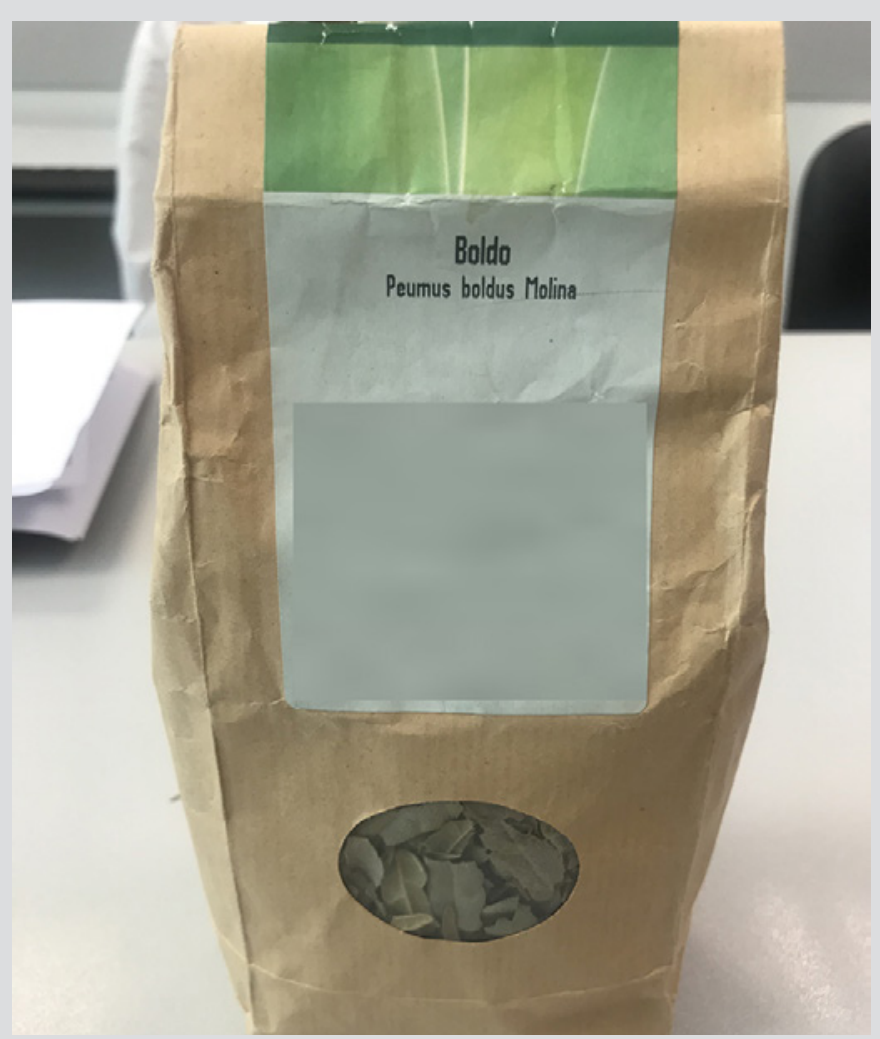

Figure 1. A packet of Peumus boldus leaves

\section{DISCUSSION}

Herbal medicines are commonly used as alternative or traditional medicines. The consumption of products of botanical origin for therapeutic purposes has increased in industrialized countries during the last few decades. A belief that natural products are harmless is strongly rooted in society, a conviction reinforced by the fact that they are prescription-free and easily obtained. Also, there are few reports in the media of their potentially harmful effects ${ }^{[1]}$.

Peumus boldus is a tree which grows in the South American Andes and is traditionally used for symptomatic treatment of dyspepsia, mild gastrointestinal spasmodic disorders and painful joints ${ }^{[2]}$. However, the number of reported cases of possible hepatotoxicity is increasing ${ }^{[1,3]}$. It is still not widely known that herbal medicines commonly cause adverse events, and their pathogenic mechanisms have not been sufficiently investigated. Cases of hepatotoxicity, the best documented and most widely studied adverse effect, have risen in recent years, in direct proportion to the increased consumption of these types of products ${ }^{[1]}$. Clinical suspicion is essential, and the patient should be queried about the consumption of non-conventional drugs.

In our case, as Peumus boldus may harm the liver, a diagnosis of toxic hepatic damage was made after the identification of a temporal correlation between exposure and the clinical symptoms and laboratory results, the exclusion of other potentially hepatotoxic factors, and the resolution of symptoms after drug cessation. The leaves of Peumus boldus contain polyphenolic compounds, including catechins. The most significant compound, quantitatively and in relation to pharmacoactivity, is epigallocatechin gallate (EGCG). There is a lack of conclusive data, but the toxic effect of catechins appears to be mediated by EGCG, which reaches variables concentrations in the final product, depending on the preparation processes used ${ }^{[1]}$. Peumus boldus leaves consumed as an infusion can be hepatotoxic and may cause otherwise unexplained jaundice or abnormal liver enzyme values, particularly in elderly patients ${ }^{[4]}$.

The safety of natural products used as alternative medicines is not properly monitored by health regulatory agencies. Their availability in non-specialized stores, their ease of acquisition without a prescription, and their easy availability on the internet, have driven the large growth in consumption seen in developed countries. The risks associated with the consumption of uncertified products circulating at the periphery of pharmacosurveillance regulations constitute a growing problem, and require effective attention by clinicians, particularly with regard to their undesirable effects. 


\section{REFERENCES}

1. Nortadas R, Barata J. Herbal therapy-induced toxic hepatitis - a case report. Revista da Sociedade Portuguesa de Medicina Interna 2011;18(2):89-92.

2. European Medicines Agency. Committee on Herbal Medicinal Products (HMPC). Assessment report on Peumus boldus Molina, folium. EMA/HMPC/453726/2016. 2 November 2016

3. Piscaglia F, Leoni S, Venturi A, Graziella F, Donati G, Bolondi L. Caution in the use of boldo in herbal laxatives: a case of hepatotoxicity. Scand J Gastroenterol 2005;40(2):236-239.

4. Ribeiro RJ, Silvestre C, Duarte C. Hidden risks of alternative medicines: a case of boldo-induced hepatotoxicity. J Diet Suppl 2017;14(2):186-190. 